\title{
AN ENERGY-AWARE SPANNING TREE AIGORITHM FOR DATA AGGREGATION IN WIRELESS SENSOR NETWORKS
}

\author{
Marc Lee and Vincent W.S. Wong \\ Department of Electrical and Computer Engineering \\ The University of British Columbia,Vancouver, BC, Canada \\ e-mail: $\{$ wnmlee, vincentw $\} @$ ece.ubc.ca
}

\begin{abstract}
In wireless sensor networks, data aggregation is used to collect local data from neighboring nodes (sources) and generate a data report. In this paper, we propose E-Span, which is an energy-aware spanning tree algorithm. E-Span is a distributed protocol and facilitates the sources within an event region to perform data aggregation. In $E$-span, the source node which has the highest residual energy is chosen as the root. Other source nodes choose their corresponding parent node among their neighbors based on the residual energy and distance to the root. By selecting the Directed Diffusion [1] as the underlying routing platform, simulation results show that the lifetime of sources can be extended significantly when $E$ Span is used.
\end{abstract}

\section{INTRODUCTION}

Wireless sensor networks (WSNs) are typically comprised of a large number of sensors being randomly deployed for detecting and monitoring tasks. These sensors, developed at a low cost and in small size (mm-scale for smart dust motes [2]), are responsible for sensing, data processing, and routing activities. Applications of such networks range from battlefield communication systems (e.g. intrusion detections and target surveillance) to environmental monitoring networks such as habitat monitoring, chemical sensing, infrastructure security, inventory and traffic control.

Since sensors are densely-deployed in WSNs, the detection of a particular stimulus or event can trigger the response from many nearby sensor nodes. Data in WSNs are usually not directly transmitted to the interested users upon event detection. Instead, nearby sensor nodes (sources) would aggregate the data locally to remove any redundancy. Thus, it is beneficial to perform data aggregation via innetwork processing to reduce the communication cost and improve the energy efficiency.

Various data aggregation algorithms are proposed in the literature. In [4], an Energy-Aware Data Aggregation Tree (EDAT) is proposed. The root periodically sends a control message. Each node upon receiving this message for the first time starts a timer. The expiration time is inversely proportional to the node's residual energy. In [5], a dynamic convoy tree-based collaboration framework for tracking a mobile target is proposed. Heuristics are used to predict the object's moving direction. A dynamic tree is then created by adding or pruning the sensors near the target.

Since the coverage area of individual sensor nodes usually overlaps, an area-dominating set is the smallest subset of nodes that covers the monitoring area. In [6], a distributed spanning tree is constructed over the areadominating set for aggregating reply messages from various event sources. In [7], a tree that is rooted at the base station is constructed such that the link cost from each node to the base station is minimized. However, the algorithm is centralized and the knowledge of global connectivity is required.

Some recent work proposed the partition of network into small clusters. Nodes which belong to the same cluster can aggregate their readings via the cluster head. The scheme in [8] periodically selects cluster heads according to a hybrid of the residual energy and node degree. However, it assumes that nodes have variable transmission power to maintain a certain degree of connectivity between the clusters.

We consider a network with $M$ randomly deployed sensors in which each node $m$ has an identical transmission range and initial residual energy $e_{m}$. An event, triggering $N$ sensors around it, occurs at a random place in the network. Data reports from these sources are clock-driven upon event detection. We define the node lifetime to be the time that a source node runs out of its energy.

In this paper, we propose E-Span, which is an energyaware spanning tree algorithm. E-Span is a distributed protocol and facilitates the sources within an event region to perform data aggregation. In E-span, the source node which has the highest residual energy is chosen as the root. Other source nodes choose their corresponding parent node among their neighbors based on the information of the residual energy and distance to the root. By selecting the Directed Diffusion [1] as the underlying routing platform, simulation results show that the lifetime of sources can be extended significantly when E-Span is used.

The remainder of this paper is organized as follows. The background of the distributed spanning tree algorithm is given in Section II. Our proposed E-Span protocol is described in Section III. Results of the performance improvement of data aggregation are presented in Section IV. Conclusions are given in Section V.

\section{BACKground: SPANNINg Tree Protocol}

A spanning tree is a graph that spans all the nodes as vertices and contains no cycles. The tree is structured in the way that the node with the smallest identifier is chosen as the root. All other nodes are connecting to this selected root via 
Define: $\quad r_{n}$ to be the ID of the root selected by node $n$ $d_{n}$ to be the shortest-path distance from $r_{n}$ to node $n$ $g_{n}=\left(n, r_{n}, d_{n}\right)$ to be the message sent by node $n$ $p_{n}$ to be the ID of the parent selected by node $n$ $t_{\text {recv, } n}$ to be the time node $n$ received the message from its parent

Initialize: $g_{n}$ to $(n, n, 0) \forall n \in N$

$p_{n}$ to $n \forall n \in N$

$t_{\text {rec }, n}$ to $O \forall n \in N$

GetSpan (node ID n, time t, timeframe T)

1 if $n$ is not an event source,

2 return

3 else \{single-hop broadcast $g_{n}$ and start a timer $P$ that expires every $T$ sec

4 while true,

5 if timer $P$ expires and $\left(r_{n}=n\right.$ or $\left.t>t_{\text {rec }, n}+T\right)$,

$6 \quad \operatorname{set}_{n}$ to $(n, n, 0)$

$7 \quad$ set $p_{n}$ to $n$

8 set $t_{\text {recv, }, \text { to } t}$

9 single-hop broadcast $g_{n}$

10 if receiving a message $g_{i}$ from node $i$,

11 if $r_{i}<r_{n}$ or $\left(r_{i}=r_{n}\right.$ and $\left.d_{i}+1<d_{n}\right)$,

$$
\text { or }\left(r_{i}=r_{n}, d_{i}+1=d_{n} \text {, and } i \leq p_{n}\right) \text {, }
$$

$12 \quad \operatorname{set}_{n}$ to $\left(n, r_{i}, d_{i}+1\right)$

$13 \quad$ set $p_{n}$ to $i$

14 set $t_{\text {recv, }, \text { to } t}$

$15 \quad$ single-hop broadcast $g_{n}$ and restart timer $\left.P\right\}$

Fig. 1 The distributed spanning tree protocol. $n$ is the node that runs the GetSpan algorithm and $n \in N$.

the shortest-path route. The protocol requires each node to exchange configuration messages in a format that contains its own identifier, its selected root, and the distance (in hops) to this selected root. Each node updates its configuration message upon identifying a root with a smaller identifier or the shortest-path neighbor. Furthermore, the neighbor for which the shortest-path configuration message comes from is chosen as the parent of a node whenever it is detected. Node identifier is used to break ties if necessary.

The GetSpan algorithm is shown in Fig 1. Single-hop broadcast refers to the operation of sending a packet to all single-hop neighbors. Lines 1 and 2 restrict the message exchanges to be within the event region. Line 3 starts the exchange and an additional timer for tree maintenance. Line 4 triggers an infinite loop. Lines 5 to 9 allow a root to periodically generate a message every $\mathrm{T}$ seconds and reset a node when it starts to lose its shortest-path neighbor. Lines 10 to 15 update the node itself and forward the message whenever a node identifies a root with a smaller identifier or a better shortest-path neighbor.

\section{Energy-AWare Spanning Tree Protocol (E-SPAN)}

E-Span is a graph that covers all the nodes as vertices and contains no cycles. All other nodes are still connected to the

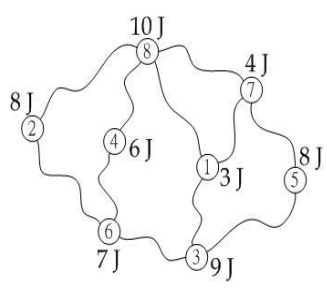

(a) Connectivity diagram

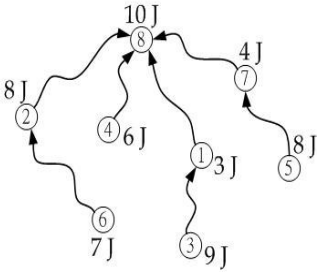

(b) E-Span configurations
Fig. 2 An example of E-Span protocol: (a) Connectivity diagram for a set of given source nodes. (b) E-Span configurations will have node 8 with energy $10 \mathrm{~J}$ chosen as the tree root.

selected root via the shortest- path route. Since the root is also responsible to coordinate the routes with distant sinks, the node with the highest energy level is now chosen as the root. Each other node is given with the choice to select its parent as the highest-energy neighbor for which the shortestpath message comes from. By using the same set of nodes as an example, the tree will now have node 8 chosen as the root and all other nodes are still talking to node 8 via the shortestpath route (see in Fig 2). Node 6 which finds itself having two shortest-path neighbors of nodes 2 and 4 will attach itself to the higher-energy one (i.e. node 2). This allows a node that has more available resources to be selected as a parent node.

The E-Span protocol is shown in Fig 3. The configuration message now involves 3 additional parameters: the residual energy of the node that sends the message, that of the node's chosen root, and the node's chosen parent. Lines 1 to 3 begin the message exchanges and restrict these exchanges to be within the event area. Lines 4 to 7 allow a root to periodically generate a message every $T$ seconds and reset a node that loses connection with its parent. Lines 8 to 11 update the list of child nodes for the receiving node. Lines 12 to 16 update the message when a node receives an energy update from its parent, or when it detects a better shortest-path neighbor or a higher-energy root. Lines 14 and 15 compare the receiving node with the root. Line 16 broadcasts the message if there is a change. Single-hop broadcast corresponds to sending a packet to all single-hop neighbors.

Our proposed E-Span has the same objective as [4]-[7] in an attempt to construct a data aggregation tree and select a dedicated root for which data are gathered. E-Span, EDAT [4], and HEED [8] consider the residual energy, thereby enhancing the chance of distributing the loads over higherenergy nodes. While the work in [4][6][7] requires the prior knowledge or support from a given tree root, E-Span does not require the root to be any particular node. In terms of root selection, we consider the residual energy of nodes within the event area whereas the work in [6][7] compares the link cost that associates with each of them.

\section{Performance Evaluation}

\section{A. Simulation Model}

We implemented our tree construction modules on top of Directed Diffusion [1] in the ns-2 [11]. A square field with each side measuring $\mathrm{L}$ meters is considered. A number of $M$ 


\section{Define:}

$e_{n}$ to be the residual energy of node $n$

$r_{n}$ to be the ID of the root selected by node $n$

$e\left(r_{n}\right)$ to be the last-updated energy of root selected by node $n$

$d_{n}$ to be the shortest-path distance from $r_{n}$ to node $n$

$p_{n}$ to be the parent selected by node $n$

$s_{n}=\left(n, e_{n}, r_{n}, e\left(r_{n}\right), p_{n}, d_{n}\right)$ to be the message sent by node $n$

$e\left(p_{n}\right)$ to be the last-updated energy of parent selected by node $n$

$t_{\text {rec }, n}$ to be the time node $n$ received the message from its parent childList $_{n}$ to be the list of child nodes for node $n$

Initialize: Change $\left(n, e_{n}, n, e_{n}, 0,0\right) \forall n \in N$

GetESpan (node ID $n$, node energy $e_{n}$, time $t$, timeframe $T$ )

1 if $n$ is not an event source,

2 return

3 else \{ single-hop broadcast $s_{n}$, start timer $P$ that expires every $T$ sec

4 while true,

5 if timer $P$ expires and $\left(r_{n}=n\right.$ or $\left.t>t_{\text {recv }, n}+T\right)$,

$6 \quad$ Change $\left(n, e_{n}, n, e_{n}, 0, t\right)$

$7 \quad$ single-hop broadcast $s_{n}$

8 if receiving a message $s_{i}$ from node $i$,

9 if $p_{i}=n$,

10 add $i$ to childList $_{n}$

11 else remove $i$ from childList $t_{n}$

$$
\left.e_{i}>e\left(p_{n}\right)\right) \text {, }
$$

$\operatorname{or}\left(d_{i}+1=d_{n}, e_{i}=e\left(p_{n}\right)\right.$, and $\left.i<p_{n}\right)$,

Change $\left(i, e_{i}, r_{i}, e\left(r_{i}\right), d_{i}+1, t\right)$

else if $\left(e\left(r_{i}\right)>e\left(r_{n}\right)\right)$ or $\left(e\left(r_{i}\right)=e\left(r_{n}\right)\right.$ and $\left.r_{i}<r_{n}\right)$,

Change $\left(i, e_{i}, r_{i}, e\left(r_{i}\right), d_{i}+1, t\right)$

if $\left(e_{n}>e\left(r_{n}\right)\right)$ or $\left(e_{n}=e\left(r_{n}\right)\right.$ and $\left.n<r_{n}\right)$,

Change $\left(n, e_{w}, n, e_{n}, 0, t\right)$

single-hop broadcast $s_{n}$ if a change applied\}

Change (node $x$, energy $e_{x}$, node $y$, energy $e_{y}$, distance $d$,

$$
\begin{array}{ll} 
& \multicolumn{1}{c}{\text { time } t)} \\
1 & \text { set } s_{n} \text { to }\left(n, e_{n}, y, e_{y}, d\right) \\
2 & \text { set } p_{n} \text { to } x \\
3 & \text { set } e\left(p_{n}\right) \text { to } e_{x} \\
4 & \text { set } t_{\text {recv, },} \text { to } t
\end{array}
$$

Fig. 3 The distributed E-Span protocol.

identical nodes, ranging from 50 to 250 in increment of 50, are randomly deployed in this field such that the average node density is kept at $\lambda=50 / 160^{2}$ nodes $/ \mathrm{m}^{2}$. Five sinks and $N$ sources are randomly chosen among the nodes, subject to the conditions that $N=0.1 M$ and sources be interconnected to each other (to model a single stimulus). The radio range is 40 meters. Each source generates random data reports of size 136 bytes with rate of 1 packet/sec. An application that computes the average of reports generated by various sources is used to model the aggregation behavior.

To reduce the simulation time, we altered the ns-2 energy model such that the sources carry an initial energy that is randomly chosen between 10 and $15 \mathrm{~J}$. All pure relaying

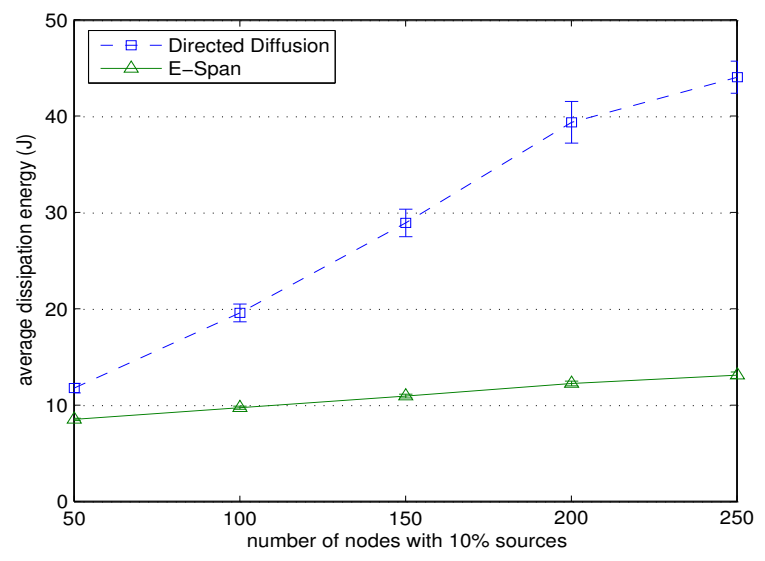

Fig. 4 Average dissipation energy as a function of network size.

nodes are given with much higher initial energy so that the performance of the lifetime of the sources can be studied. Idle time power, receive power, and transmit power dissipation are set at 35, 395, $660 \mathrm{~mW}$, respectively. The data processing and aggregation cost are assumed to be negligible.

\section{B. Results and Discussions}

We first determine the average dissipated energy, which is defined as the average amount of energy consumed by a relaying sensor node throughout the entire simulation. This metric is used to study the impact of aggregation on energy reduction. Results are averaged over 20 different runs with a 95\% confidence interval. Results in Fig 4 show significant energy reduction by using E-Span when compared with the Directed Diffusion. This shows the benefits of data aggregation when data from various neighboring sources are summarized into a single message.

We use the metric average node lifetime to measure the time at which a source runs out of its available energy. Fig 5 shows the results when $M=250$ (similar results for other network sizes can be found in [10]). By combining reports from various sources, E-Span reduces a considerable amount of redundant traffic in the network. Since less energy is being consumed, there is a lifetime improvement when data are collected and aggregated via the trees.

Fig 6 shows the average data packet delay between each source and sink. Results show that the E-Span incurs a lower average delay than the Directed Diffusion. Since E-Span aggregates data from various sources, it is as if only a single source is generating packets. This is also true in a network with a large number of data sources.

Fig 7 shows the average packet delivery ratio for Directed Diffusion and E-Span. Results show that E-Span is able to maintain a relatively high packet delivery ratio even when $N$ increases. The Directed Diffusion has its network overloaded with data traffic when more sources are sending packets. Some data packets are therefore being dropped. Since E-Span transmits data to the network as if there is only a single source, it can maintain a higher packet delivery ratio. 


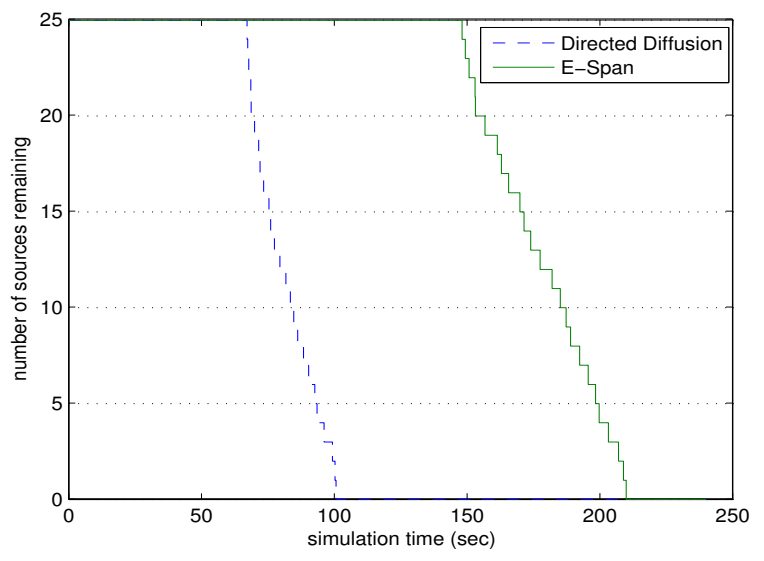

Fig. 5 Average node lifetime for each source with $M=250$.

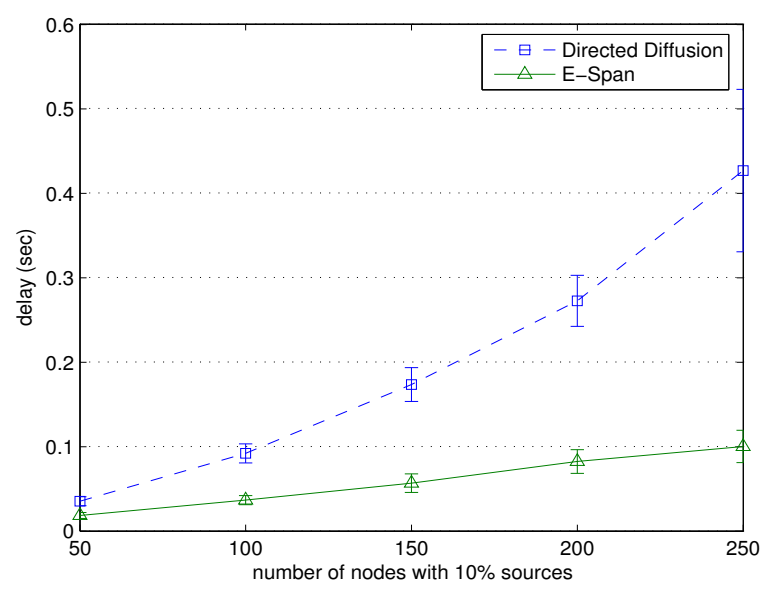

Fig. 6 Average data packet delay between a source and sink.

\section{Conclusions}

In this paper, we proposed E-Span, which is an energyaware spanning tree algorithm. E-Span is a distributed protocol and facilitates the sources within an event region to perform data aggregation. In E-span, the source node which has the highest residual energy is chosen as the root. Each other node chooses its parent node among its neighbors based on the information of the residual energy and distance to the root. Simulation results show that the average node lifetime for sensors using E-Span is higher than that of the Directed Diffusion. E-Span also maintains a low average packet transfer delay and a high packet delivery ratio. Further work includes performance comparison between E-Span and other data aggregation algorithms.

\section{ACKNOWLEDGEMENT}

This work was supported by the Natural Sciences and Engineering Research Council of Canada (NSERC) under grant number 261604-03.

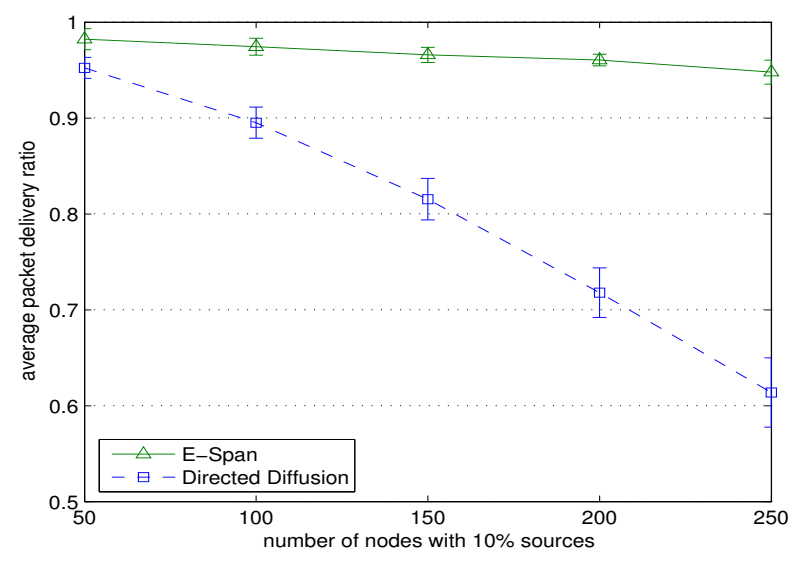

Fig. 7 Average packet delivery ratio between transmitting a data packet from each source and receiving it at each sink.

\section{REFERENCES}

[1] C. Intanagonwiwat, R. Govindan, and D. Estrin, "Directed diffusion: A scalable and robust communication paradigm for sensor networks," in Proc. of ACM MobiCom'00, Boston, MA, Aug. 2000.

[2] J. M. Kahn, R. H. Katz, and K. S. J. Pister, "Next century challenges: Mobile networking for smart dust," in Proc. of ACM MobiCom'99, Seattle, WA, Aug. 1999.

[3] G. J. Pottie and W. J. Kaiser, "Wireless integrated network sensors," Communications of the ACM, vol. 43, no. 5, pp. 5158, May 2000.

[4] M. Ding, X. Cheng, and G. Xue, "Aggregation tree construction in sensor networks," in Proc. of IEEE VTC'03, vol. 4, Orlando, FL, Oct. 2003.

[5] W. Zhang and G. Cao, "DCTC: Dynamic convoy tree-based collaboration for target tracking in sensor networks," IEEE Trans. on Wireless Communications, vol. 3, no. 5, pp. 16891701, Sept. 2004.

[6] J. Carle and D. Simplot-Ryl, "Energy-efficient area monitoring for sensor networks," IEEE Computer Magazine, vol. 37, no. 2, pp. 40-46, Feb. 2004.

[7] S. Upadhyayula, V. Annamalai, and S. K. S. Gupta, "A lowlatency and energy-efficient algorithm for convergecast," in Proc. of IEEE GLOBECOM'03, Dec. 2003.

[8] O. Younis and S. Fahmy, "HEED: A hybrid, energy-efficient, distributed clustering approach for ad hoc sensor networks," IEEE Trans. on Mobile Computing, vol. 3, no. 4, pp. 366-379, Oct. 2004.

[9] Y. K. Dalal and R. M. Metcalfe, "Reverse-path forwarding of broadcast packets," Communications of the ACM, vol. 21, no. 12, pp. 1040-1048, Dec. 1978.

[10] W.N.M. Lee, "The construction of a lifetime-preserving tree for data aggregation in wireless sensor networks," M.A.Sc. Thesis, University of British Columbia, Canada, Nov. 2004.

[11] VINT. (2001, Nov.) The network simulator ns-2. [Online]. Available: http://www.isi.edu/nsnam/ns. 